\title{
Various Approaches for Solving the Network Problems using $\lambda$ TLR Intuitionistic Fuzzy Numbers
}

\author{
P. Jayagowri \\ Assistant professor \\ Department of Mathematics, \\ Sudharsan Engineering college, \\ Pudukottai,Tamilnadu, India
}

\author{
G. Geetharamani, PhD. \\ Assistant professor \\ Department of Mathematics, \\ Anna University of Technology, \\ Tiruchirappalli, Tamilnadu, India.
}

\begin{abstract}
The shortest path problem is an important classical network optimization problem which has wide range of application in various fields.In this paper, algorithms are proposed for the Intuitionistic fuzzy shortest path problem, where the arc length of the network takes imprecise numbers, instead of real numbers namely level $\lambda$-triangular LR fuzzy numbers $(\lambda-$ TLR). Finally an illustrative example is also including demonstrating our proposed algorithm.
\end{abstract}

\section{Keywords}

A cyclic network, Shortest path problem, Level $\lambda$-TLR fuzzy numbers, Level $\lambda$-TLR indices, Signed distance of $\lambda$ - triangular fuzzy numbers.

\section{INTRODUCTION}

Many fields deal daily with the uncertain data that may not be successfully modeled by the classical mathematics. There are some mathematical tools for dealing with uncertainties. Fuzzy set theory is a powerful tool to model imprecise and vague situations where exact analysis is either difficult or impossible.

Many researchers have focused on Fuzzy Shortest Path Problem in a network since it is important to many applications such as communications, routing and transportation etc. The fuzzy set theory was introduced by Zadeh. Atanassov generalized this idea to intuitionistic fuzzy sets,and later there has been much progress in the study of intuitionistic fuzzy sets(IFSs)The fuzzy shortest path problem was first analyzed by Dubois and Prade[1]. According to their approach, the shortest path length can be obtained but the corresponding path in the network may not exist. Chuang and kung[3] proposed a fuzzy shortest path length procedure that can find a fuzzy shortest path length among all possible paths in a network. Lin and Okada and soper [4]proposed a fuzzy algorithm, which was based on multiple labeling methods to offer non-dominated paths to a decision maker.

Nayeem and $\mathrm{Pal}[7]$ has proposed an algorithm based on the Acceptability index .S.Elizabeth and L.Sujatha[8] discussed about fuzzy shortest path problem based on level $\lambda$ - TLR fuzzy numbers. Iraj Mahadavi,Ali Tajdin,Rahele Nourifar [10] discussed about FuzzyOptimization Approach for Shortest Path Problem in Network flow.

Chen[14] found the fuzzy shortest path length in a network by means of a fuzzy linear programming approach. Kiran Yadav and Ranjit Biswas [17] has proposed An Approach to Find k

th Shortest Path Using Fuzzy Logic., Sujatha ,L. and Sattanathan ,R. [21] were discussed about Fuzzy Shortest path problem based on similarity degree using LR type fuzzy numbers.

This paper is organized as follows. In section 2 some elementary concepts and operations of fuzzy set theory have been reviewed. In section 3 we introduced new indices have been defined for level $\lambda$-TLR fuzzy numbers. In section 4 , new algorithms have been proposed for Intuitionistic Fuzzy Shortest Path Problem based on $\lambda$-TLR indices. In section 5 illustrative examples are given to demonstrate to our proposed algorithm. In section 6 and 7comparative study was made finally in section 8 some conclusions are drawn..

\section{PRELIMINARIES}

In this section some basic definitions are reviewed.

\subsection{Acyclic Digraph}

A digraph is a graph each of whose edges are directed. Hence an acyclic digraph is a directed graph with out cycle.

\subsection{Level $\lambda$ - TLR fuzzy number} Level $\lambda$-TLR fuzzy number is represented by $\mathrm{A}=(\mathrm{a}, \mathrm{b}, \mathrm{c} ; \lambda)_{\mathrm{LR}}$ with the membership function

$$
\mu_{A}(x)=\left\{\begin{array}{l}
0 \\
\frac{\lambda[x-(a-b)]}{b} \\
\frac{\lambda[(a+c)-x]}{c} \\
0
\end{array}\right.
$$

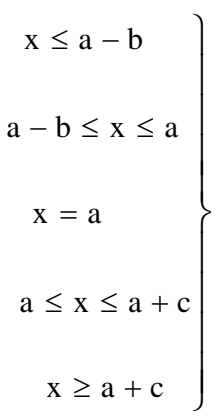

Here $\mathrm{a}$ is the point whose membership value is $\lambda$, where $0<\lambda \leq 1$ and b, c are the left hand and right hand spreads respectively. 
2.3 Minimum operation on level $\lambda$ - TLR fuzzy numbers

Let

$\mathrm{L}_{1}=\left(\mathrm{a}_{1}, \mathrm{~b}_{1}, \mathrm{c}_{1} ; \lambda\right)_{\mathrm{LR}}$ and $\mathrm{L}_{2}=\left(\mathrm{a}_{2}, \mathrm{~b}_{2}, \mathrm{c}_{2} ; \lambda\right)_{\mathrm{LR}}$ be the two level $\lambda-\mathrm{T}$ LR fuzzy numbers. Then $\mathrm{L}_{\text {min }}=\min \left(\mathrm{L}_{1}, \mathrm{~L}_{2}\right)$ $=\left(\min \left(\mathrm{a}_{1}, \mathrm{a}_{2}\right), \max \left(\mathrm{b}_{1}, \mathrm{~b}_{2}\right), \min \left(\mathrm{c}_{1}, \mathrm{c}_{2}\right) ; \lambda\right)_{L R}$

\subsection{Addition operation on Level $\lambda$ - T LR fuzzy numbers}

$\mathrm{L}_{1}=\left(\mathrm{a}_{1}, \mathrm{~b}_{1}, \mathrm{c}_{1} ; \lambda\right)_{\mathrm{LR}}$ and $\mathrm{L}_{2}=\left(\mathrm{a}_{2}, \mathrm{~b}_{2}, \mathrm{c}_{2} ; \lambda\right)_{\mathrm{LR}}$ be the two level $\lambda-\mathrm{T}$ LR fuzzy numbers. Then. $\mathrm{L}_{1}+\mathrm{L}_{2}=\left(\mathrm{a}_{1}, \mathrm{~b}_{1}, \mathrm{c}_{1} ; \lambda\right)_{\mathrm{LR}}+\left(\mathrm{a}_{2}, \mathrm{~b}_{2}, \mathrm{c}_{2} ; \lambda\right)_{\mathrm{LR}}$ $=\left(\mathrm{a}_{1}+\mathrm{a}_{2}, \mathrm{~b}_{1}+\mathrm{b}_{2}, \mathrm{c}_{1}+\mathrm{c}_{2} ; \lambda\right)_{\mathrm{LR}}$

\subsection{The Signed distance}

Let $d(b, 0)=b$ when, $b, 0$ belongs to $R$, Where $\mathrm{d}(\mathrm{b}, 0)$ means the signed distance of $\mathrm{b}$ measuring form 0 .

\subsection{Signed distance of level $\lambda$-TLR Fuzzy} number

For each $\lambda \varepsilon(0,1]$ and $\mathrm{A}=(\mathrm{a}, \mathrm{b}, \mathrm{c} ; \lambda)_{\mathrm{LR}}$

The signed distance from 0 to $\mathrm{A}$ is defined by $\mathrm{d}(\mathrm{A}, 0)=$ $\frac{1}{-}(2 b+a+c)$ The ranking of level $\lambda$-triangular fuzzy

numbers is defined by $\mathrm{A}<\mathrm{B}$ iff $\mathrm{d}(\mathrm{A}, 0)<\mathrm{d}(\mathrm{B}, 0)$.

The signed distance from 0 to $\mathrm{L}_{1}=\left(\mathrm{a}_{1}, \mathrm{~b}_{1}, \mathrm{c}_{1} ; \lambda\right)_{\mathrm{LR}}$ and it is denoted by

$\mathrm{d}\left(\mathrm{L}_{1}, 0\right)=\frac{1}{4}\left[2 \mathrm{a}_{1}+\left(\mathrm{a}_{1}-\mathrm{b}_{1}\right)+\left(\mathrm{a}_{1}+\mathrm{c}_{1}\right)\right]$ here

$\mathrm{L}_{1}<\mathrm{L}_{2}$ iff $\mathrm{d}\left(\mathrm{L}_{1}, 0\right)<\mathrm{d}\left(\mathrm{L}_{2}, 0\right)$

\subsection{Indices based on $\mathrm{L}_{\mathrm{i}}$ and $\mathrm{L}_{\text {min }}$}

Let the $\lambda$ - triangular LR $i^{\text {th }}$ fuzzy path length be $\mathrm{L}_{\mathrm{i}}=\left(\mathrm{a}_{\mathrm{i}}, \mathrm{b}_{\mathrm{i}}, \mathrm{c}_{\mathrm{i}} ; \lambda\right)_{\mathrm{LR}}$ and the level

$\lambda$-TLR fuzzy Shortest length be $\mathrm{L}_{\min }=(\mathrm{a}, \mathrm{b}, \mathrm{c} ; \lambda)_{\mathrm{LR}}$,

$0<\lambda \leq 1$ where $\mathrm{a}-\mathrm{b} \leq \mathrm{a}_{\mathrm{i}}-\mathrm{b}_{\mathrm{i}}, \mathrm{a} \leq \mathrm{a}_{\mathrm{i}}$,

$a+c \leq a_{i}+c_{i}$ then
The level $\lambda$ - triangular LR weighted average index between

$\mathrm{L}_{\mathrm{i}}$ and $\mathrm{L}_{\min }$ is calculated as

$\mathrm{Z}_{\mathrm{i}}^{*}\left(\mathrm{~L}_{\min ,} \mathrm{L}_{\mathrm{i}}\right)=\frac{\lambda(\mathrm{a})+\lambda\left(\mathrm{a}_{\mathrm{i}}\right)}{2 \lambda}$ here we have

$\mathrm{L}_{1}<\mathrm{L}_{2}$ iff $\mathrm{Z}_{1}^{*}\left(\mathrm{~L}_{\min ,} \mathrm{L}_{1}\right)<\mathrm{Z}_{2}^{*}\left(\mathrm{~L}_{\min ,} \mathrm{L}_{2}\right)$

\subsection{Indices based on $\mathrm{L}_{\mathrm{i}}$}

Let the $\lambda-\mathrm{T}$ LR $i^{\text {th }}$ fuzzy path length be

$\mathrm{L}_{\mathrm{i}}=\left(\mathrm{a}_{\mathrm{i}}, \mathrm{b}_{\mathrm{i}}, \mathrm{c}_{\mathrm{i}} ; \lambda\right)_{\mathrm{LR}}$ then

a) The level $\lambda$ - TLR mean Index $L_{i}$ is calculated as

$$
\mathrm{T}_{\text {mean }}=\frac{2 \mathrm{a}_{\mathrm{i}}+\mathrm{c}_{\mathrm{i}}-\mathrm{b}_{\mathrm{i}}}{2}
$$

b)

The level $\lambda-$ TLR centroid Index $L_{i}$

is calculated as

$$
\mathrm{T}_{\text {centroid }}\left(\mathrm{L}_{\mathrm{i}}\right)=\frac{3 \mathrm{a}_{\mathrm{i}}+\mathrm{c}_{\mathrm{i}}-\mathrm{b}_{\mathrm{i}}}{3}
$$

\section{DEFINITION}

In this paper we introduce the following definition

\subsection{Addition operation on Level $\lambda$-T LR Intuitionistic fuzzy numbers}

Let

$\mathrm{L}_{1}=\left(\mathrm{a}_{\mu 1}, \mathrm{~b}_{\mu 1}, \mathrm{c}_{\mu 1} ; \lambda\right)_{\mathrm{LR}}$ and $\mathrm{L}_{2}=\left(\mathrm{a}_{\mu 2}, \mathrm{~b}_{\mu 2}, \mathrm{c}_{\mu 2} ; \lambda\right)_{\mathrm{LR}}$

the membership function of two level $\lambda$ - TLR intuitionistic fuzzy numbers

Then

$$
\begin{aligned}
\mathrm{L}_{\text {min }} & =\min \left(\mathrm{L}_{1}, \mathrm{~L}_{2}\right) \\
= & \left.\left(\min _{\mu 1}, \mathrm{a}_{\mu 2}\right), \max \left(\mathrm{b}_{\mu 1}, \mathrm{~b}_{\mu 2}\right), \min \left(\mathrm{c}_{\mu 1}, \mathrm{c}_{\mu 2}\right) ; \lambda\right)_{L R}
\end{aligned}
$$

and

$\mathrm{L}_{1}=\left(\mathrm{a}_{\gamma 1}, \mathrm{~b}_{\gamma 1}, \mathrm{c}_{\gamma 1} ; \lambda\right)_{\mathrm{LR}}$ and $\mathrm{L}_{2}=\left(\mathrm{a}_{\gamma 2}, \mathrm{~b}_{\gamma 2}, \mathrm{c}_{\gamma 2} ; \lambda\right)_{\mathrm{LR}}$ be the Non membership function of two level $\lambda$ - TLR 


$$
\begin{array}{ll}
\text { intuitionistic fuzzy } & \text { numbers. } \\
\mathrm{L}_{\max }=\max \left(\mathrm{L}_{1}, \mathrm{~L}_{2}\right) & \\
=\left(\max \left(\mathrm{a}_{\gamma 1}, \mathrm{a}_{\gamma 2}\right), \min \left(\mathrm{b}_{\gamma 1}, \mathrm{~b}_{\gamma 2}\right), \max \left(\mathrm{c}_{\gamma 1}, \mathrm{c}_{\gamma 2}\right) ; \lambda\right)_{\mathrm{LR}}
\end{array}
$$

\subsection{Weighted Average Index for $\lambda$ TLR number (Membership function)}

Let the level $\lambda$ TLR $i^{\text {th }}$ fuzzy path length be $\mathrm{L}_{\mathrm{i}}=\left(\mathrm{a}_{\mu \mathrm{i}}, \mathrm{b}_{\mu \mathrm{i}}, \mathrm{c}_{\mu \mathrm{i}} ; \lambda\right)_{\mathrm{LR}}$ and the level $\lambda$ TLR Intuitionistic fuzzy shortest length be $\mathrm{L}_{\text {min }}=\left(\mathrm{a}_{\mu}, \mathrm{b}_{\mu}, \mathrm{c}_{\mu} ; \lambda\right)_{\mathrm{LR}} \quad 0<\lambda \leq 1 \quad$ where $a_{\mu}-b_{\mu} \leq a_{\mu i}-b_{\mu i}, a_{\mu} \leq a_{\mu i}$, $\mathrm{a}_{\mu}+\mathrm{c}_{\mu} \leq \mathrm{a}_{\mu_{\mathrm{i}}}+\mathrm{c}_{\mu_{\mathrm{i}}}$ then the level $\lambda$ triangular LR weighted average index between $\mathrm{L}_{\text {min }}$ and $\mathrm{L}_{\mathrm{i}}$ is calculated as $\mathrm{Z}_{\mathrm{i}}^{*}\left(\mathrm{~L}_{\min }, \mathrm{L}_{\mathrm{i}}\right)=\frac{\lambda \mathrm{a}_{\mu}+\lambda \mathrm{a}_{\mu}{ }_{\mathrm{i}}}{2}$ herewehave $\mathrm{L}_{1}<\mathrm{L}_{2}$ iff $\mathrm{Z}_{1}^{*}\left(\mathrm{~L}_{\min }, \mathrm{L}_{1}\right)<\mathrm{Z}_{2}^{*}\left(\mathrm{~L}_{\min }, \mathrm{L}_{2}\right)$

\subsection{Weighted Average Index for $\lambda$ TLR} number (Non Membership function)

Let the level $\lambda$ TLR $i^{\text {th }}$ fuzzy path length be $\mathrm{L}_{\mathrm{i}}=\left(\mathrm{a}_{\gamma \mathrm{i}}, \mathrm{b}_{\gamma \mathrm{i}}, \mathrm{c}_{\gamma \mathrm{i}} ; \lambda\right)_{\mathrm{LR}}$ and the level $\lambda$ TLR fuzzy shortest length be $\mathrm{L}_{\max }=\left(\mathrm{a}_{\gamma}, \mathrm{b}_{\gamma}, \mathrm{c}_{\gamma} ; \lambda\right)_{\mathrm{LR}} \quad 0<\lambda \leq 1$

where

$\mathrm{a}_{\gamma}-\mathrm{b}_{\gamma} \leq \mathrm{a}_{\gamma \mathrm{i}}-\mathrm{b}_{\gamma \mathrm{i}}, \mathrm{a}_{\gamma} \leq \mathrm{a}_{\gamma \mathrm{i}}, \mathrm{a}_{\gamma}+\mathrm{c}_{\gamma} \leq \mathrm{a}_{\gamma_{\mathrm{i}}}+\mathrm{c}_{\gamma_{\mathrm{i}}}$ then the level $\lambda$ TLR weighted average index between $\mathrm{L}_{\max }$ and $\mathrm{L}_{\mathrm{i}}$ is calculated as

$\mathrm{Z}_{\mathrm{i}}^{*}\left(\mathrm{~L}_{\max }, \mathrm{L}_{\mathrm{i}}\right)=\frac{{ }^{\lambda \mathrm{a}}+\lambda \mathrm{a}_{\mathrm{i}}}{2}$ here we have $\mathrm{L}_{1}<\mathrm{L}_{2}$ iff $\mathrm{Z}_{1}^{*}\left(\mathrm{~L}_{\max }, \mathrm{L}_{1}\right)<\mathrm{Z}_{2}^{*}\left(\mathrm{~L}_{\max }, \mathrm{L}_{2}\right)$

\subsection{Indices based on $L_{i}$ for membership}

\section{function}

Let the $\lambda-$ TLR $i^{\text {th }}$ Intuitionistic fuzzy

path length be $\mathrm{L}_{\mu \mathrm{i}}=\left(\mathrm{a}_{\mu \mathrm{i}}, \mathrm{b}_{\mu \mathrm{i}}, \mathrm{c}_{\mu \mathrm{i}} ; \lambda\right)_{\mathrm{LR}}$ then

1) The level $\lambda$-triangular LR mean index for

$$
\mathrm{L}_{\mu \mathrm{i}} \text { is calculated as } \mathrm{T}_{\text {Mean }}=\frac{2 \mathrm{a}_{\mu \mathrm{i}}+\mathrm{c}_{\mu \mathrm{i}}-\mathrm{b}_{\mu \mathrm{i}}}{2}
$$

2) The level $\lambda$-triangular LR centroid index for $L_{\mu i}$ is calculated as $\quad \mathrm{T}_{\text {centroid }}=\frac{3 \mathrm{a}_{\mu \mathrm{i}}+\mathrm{c}_{\mu \mathrm{i}}-\mathrm{b}_{\mu \mathrm{i}}}{3}$

\subsection{Indices based on $L_{i}$ for Non}

\section{membership function}

Let the $\lambda$-TLR $\mathrm{i}^{\text {th }}$ Intuitionistic path length be $\mathrm{L}_{\gamma \mathrm{i}}=\left(\mathrm{a}_{\gamma \mathrm{i}}, \mathrm{b}_{\gamma \mathrm{i}}, \mathrm{c}_{\gamma \mathrm{i}} ; \lambda\right){ }_{\mathrm{LR}}$ then

1) The level $\lambda-T$ LR mean index for

$$
\mathrm{L}_{\gamma \mathrm{i}} \text { is calculated as } \mathrm{T}_{\text {Mean }}=\frac{{ }_{\gamma \mathrm{i}} \mathrm{a}_{\gamma \mathrm{i}}-\mathrm{c}_{\gamma \mathrm{i}}}{2}
$$

2) The level $\lambda$-triangular LR centroid index for $\mathrm{L}_{\gamma \mathrm{i}}$ is calculated as $\mathrm{T}_{\text {centroid }}=\frac{3 \mathrm{a} \gamma \mathrm{i}+\mathrm{b}_{\gamma \mathrm{i}}-\mathrm{c} \gamma \mathrm{i}}{3}$

\subsection{Signed distance of level $\lambda$-T LR} Intuitionistic Fuzzy number(Membership Function)

For each $\lambda \varepsilon(0,1]$ and $\mathrm{A}=(\mathrm{a}, \mathrm{b}, \mathrm{c} ; \lambda) \mathrm{LR}$

The signed distance from 0 to $\mathrm{A}$ is defined by $\mathrm{d}(\mathrm{A}, 0)=\frac{1}{4}(2 \mathrm{~b}+\mathrm{a}+\mathrm{c})$ The ranking of level $\lambda-$ triangular Intuitionistic fuzzy numbers is defined by $A<B$ iff $d(A, 0)<d(B, 0)$.

The signed distance from 0 to $\mathrm{L}_{1}=\left(\mathrm{a}_{\mu_{1}}, \mathrm{~b} \mu_{1}, \mathrm{c} \mu_{\mu_{1}} ; \lambda\right)_{\mathrm{LR}}$ and it is denoted by $\mathrm{d}\left(\mathrm{L}_{1}, 0\right)=\frac{1}{4}\left[2 \mathrm{a}_{\mu_{1}}+\left(\mathrm{a}_{\mu_{1}}-\mathrm{b}_{\mu_{1}}\right)+\left(\mathrm{a}_{\mu_{1}}+\mathrm{c}_{\mu_{1}}\right)\right]$ here $\mathrm{L}_{1}<\mathrm{L}_{2}$ iff $\mathrm{d}\left(\mathrm{L}_{1}, 0\right)<\mathrm{d}\left(\mathrm{L}_{2}, 0\right)$

3.7 Signed distance of level $\lambda$ - T LR Intuitionistic Fuzzy number(Non Membership Function)

For each $\lambda \varepsilon(0,1]$ and $\mathrm{A}=(\mathrm{a}, \mathrm{b}, \mathrm{c} ; \lambda){ }_{\mathrm{LR}}$ 
The signed distance from 0 to $\mathrm{A}$ is defined by

$\mathrm{d}(\mathrm{A}, 0)=\frac{1}{4}(2 \mathrm{~b}+\mathrm{a}+\mathrm{c})$ The ranking of level $\lambda-$ triangular Intuitionistic fuzzy numbers is defined by $\mathrm{A}<\mathrm{B}$ iff $\mathrm{d}(\mathrm{A}, 0)<\mathrm{d}(\mathrm{B}, 0)$

The signed distance from 0 to $\mathrm{L}_{1}=\left(\mathrm{a}_{\gamma_{1}}, \mathrm{~b}_{\gamma_{1}}, \mathrm{c}{ }_{\gamma_{1}} ; \lambda\right)_{\mathrm{LR}}$ and it is denoted by $\mathrm{d}\left(\mathrm{L}_{1}, 0\right)=\frac{1}{4}\left[2 \mathrm{a}_{\gamma_{1}}+\left(\mathrm{b}_{\mu_{1}}-\mathrm{a}_{\mu_{1}}\right)+\left(\mathrm{a}_{\mu_{1}}-\mathrm{c}_{\mu_{1}}\right)\right]$ here $\mathrm{L}_{1}<\mathrm{L}_{2}$ iff $\mathrm{d}\left(\mathrm{L}_{1}, 0\right)<\mathrm{d}\left(\mathrm{L}_{2}, 0\right)$

\section{PROPOSED ALGORITHM}

\subsection{Proposed Algorithm for Intuitionistic} Fuzzy Shortest Path Problem Based On Level $\lambda$ - TLR Intuitionistic Fuzzy Number In this paper we introduce a new method called 'weighted index' which is the measurement tool between $\mathrm{L}_{\min }$ and $\mathrm{L}_{\mathrm{i}}$ Let $\mathrm{L}_{\mathrm{i}}=\left(\mathrm{a}_{\mu \mathrm{i}}, \mathrm{b}_{\mu \mathrm{i}}, \mathrm{c}_{\mu \mathrm{i}} ; \lambda\right){ }_{\mathrm{LR}}$ and

$$
\mathrm{L}_{\mathrm{i}}=\left(\mathrm{a}_{\gamma \mathrm{i}}, \mathrm{b}_{\gamma \mathrm{i}}, \mathrm{c}_{\gamma \mathrm{i}} ; \lambda\right)_{\mathrm{LR}} \quad \mathrm{i}=1,2, \ldots \mathrm{n} \text { where }
$$

$\mathrm{L}_{\mathrm{i}}$ denotes the level $\lambda_{-} \mathrm{T}$ LR Intuitionistic fuzzy path length.

Step 1 Construct a network $G=(V, E)$ Which is an acyclic digraph and the arc length takes the level $\lambda$ - TLR intuitionistic fuzzy numbers.

Step 2 Calculate all possible path $P_{i}$ from source node to destination node and compute the corresponding path length $\mathrm{L}_{\mathrm{i}} \mathrm{i}=1,2, \ldots \mathrm{m}$ for possible $\mathrm{m}$ path using definition and set $\mathrm{L}_{\mathrm{i}}=\left(\mathrm{a}_{\mu \mathrm{i}}, \mathrm{b}_{\mu \mathrm{i}}, \mathrm{c}_{\mu \mathrm{i}} ; \lambda\right)_{\mathrm{LR}}$ and $L_{i}=\left(a_{\gamma i}, b_{\gamma i}, c_{\gamma i} ; \lambda \lambda L R\right.$ Where $\mathrm{i}=1$ to $\mathrm{n}$, for Membership function and Nonmembeship function respectively also $0<\lambda \leq 1$

Step 3 Calculate the Intuitionistic fuzzy shortest length $\mathrm{L}_{\text {min }}$ and $\mathrm{L}_{\max }$ using definition 3.1 and set $\mathrm{L}_{\text {min }}=\left(\mathrm{a}_{\mu}, \mathrm{b}_{\mu}, \mathrm{c}_{\mu} ; \lambda\right)_{\mathrm{LR}} \quad \mathrm{L}_{\max }=\left(\mathrm{a}_{\gamma}, \mathrm{b}_{\gamma}, \mathrm{c}_{\gamma} ; \lambda\right)_{\mathrm{LR}}$ Step 4 Calculate the level $\lambda$-TLR intuitionistic fuzzy weighted average between $\mathrm{L}_{\min }, \mathrm{L}_{\max }$ and $\mathrm{L}_{\mathrm{i}}$
Using definition 3.2 and 3.3 for $\mathrm{i}=1$ to $\mathrm{n}$.

Step 5 The path having the Minimum level $\lambda$ TLR intuitionistic fuzzy weighted average is identified as the shortest path.

\subsection{Proposed Algorithm for Intuitionistic} Fuzzy Shortest Path Problem Based on Level $\lambda$ - TLR Mean and Centroid

$$
\begin{aligned}
& \text { Let } \mathrm{L}_{\mathrm{i}}=\left(\mathrm{a}_{\mu \mathrm{i}}, \mathrm{b}_{\mu \mathrm{i}}, \mathrm{c}_{\mu \mathrm{i}} ; \lambda\right)_{\mathrm{LR}} \text { and } \\
& \mathrm{L}_{\mathrm{i}}=\left(\mathrm{a}_{\gamma \mathrm{i}}, \mathrm{b}_{\gamma \mathrm{i}}, \mathrm{c}_{\gamma \mathrm{i}} ; \lambda\right)_{\mathrm{LR}} \quad \mathrm{i}=1,2, \ldots \text { n where }
\end{aligned}
$$

$\mathrm{L}_{\mathrm{i}}$ denotes the level $\lambda \mathrm{T}$ LR Intuitionistic fuzzy path length.

Step 1 Construct a network $G=(V, E)$ Which is an acyclic digraph and the arc length takes the level $\lambda$ - TLR intuitionistic fuzzy numbers.

Step2 Calculate all possible path $\mathrm{P}_{\mathrm{i}}$ from source node to destination node and compute the corresponding path length $\mathrm{L}_{\mathrm{i}} \mathrm{i}=1,2, \ldots \mathrm{m}$ for possible $\mathrm{m}$ path using definition and set $\mathrm{L}_{\mathrm{i}}=\left(\mathrm{a}_{\mu \mathrm{i}}, \mathrm{b}_{\mu \mathrm{i}}, \mathrm{c}_{\mu \mathrm{i}} ; \lambda\right)_{\mathrm{LR}}$ and

$\mathrm{L}_{\mathrm{i}}=\left(\mathrm{a}_{\gamma \mathrm{i}}, \mathrm{b}_{\gamma \mathrm{i}}, \mathrm{c}_{\gamma \mathrm{i}} ; \lambda\right) \mathrm{LR}$

where $\mathrm{i}=1$ to $\mathrm{n}$, for Membership function

and Nonmembeship function respectively also

$0<\lambda \leq 1$

Step 3 Calculate the level $\lambda$-TLR mean and centroid,

Using definition3.4 and 3.5 for $\mathrm{i}=1$ to $\mathrm{n}$

Step 4 The path having the minimum Mean and Centroid is identified as the shortest path.

\subsection{Proposed Algorithm For Intuitionistic Fuzzy Shortest path problem based on signed distance}

In this paper we introduce a new method called Signed distance Let $\mathrm{L}_{\mathrm{i}}=\left(\mathrm{a}_{\mu \mathrm{i}}, \mathrm{b}_{\mu \mathrm{i}}, \mathrm{c}_{\mu \mathrm{i}} ; \lambda\right)_{\mathrm{LR}}$ and

$$
\mathrm{L}_{\mathrm{i}}=\left(\mathrm{a}_{\gamma \mathrm{i}}, \mathrm{b}_{\gamma \mathrm{i}}, \mathrm{c}_{\gamma \mathrm{i}} ; \lambda\right)_{\mathrm{LR}} \quad \mathrm{i}=1,2, \ldots \mathrm{n} \text { where }
$$


$L_{i}$ denotes the level $\lambda_{-}$T LR Intuitionistic fuzzy path length.

Step 1 Construct a network $G=(V, E)$ Where $V$ is the set of vertices and $\mathrm{E}$ is the set of edges. Here $\mathrm{G}$ is an acyclic digraph and the arc length takes the level $\lambda-\mathrm{T}$ LR intuitionistic fuzzy numbers.

Step 2 Calculate all possible path $P_{i}$ from source node to destination node and compute the corresponding path length $\mathrm{L}_{\mathrm{i}} \mathrm{i}=1,2, \ldots \mathrm{m}$ for possible $\mathrm{m}$ path using definition and set $\mathrm{L}_{\mathrm{i}}=\left(\mathrm{a}_{\mu \mathrm{i}}, \mathrm{b}_{\mu \mathrm{i}}, \mathrm{c}_{\mu \mathrm{i}} ; \lambda\right)_{\mathrm{LR}}$ and

$\mathrm{L}_{\mathrm{i}}=\left(\mathrm{a}_{\gamma \mathrm{i}}, \mathrm{b}_{\gamma \mathrm{i}}, \mathrm{c}_{\gamma \mathrm{i}} ; \lambda\right)_{\mathrm{LR}}$

$\mathrm{i}=1$ to $\mathrm{n}$, for Membership function and Nonmembeship function respectively also

$0<\lambda \leq 1$

Step 3 calculate signed distance of level $\lambda$ - TLR type representation of fuzzy interval for each possible paths using definition $3.6 \& 3.7$

Step 4 The path having the minimum signed distance of level $\lambda$ - TLR type representation of intuitionistic fuzzy interval is identified as the shortest path.

\section{ILLUSTRATIVE EXAMPLE 1}

Step1 Construct a network with 6 vertices and 8 edges as follows

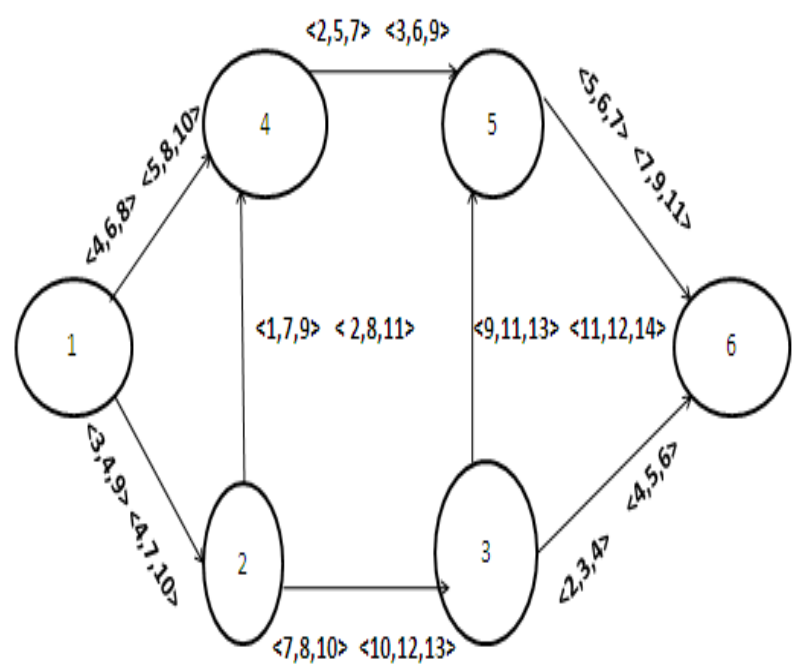

Let $\lambda=1$

Step 2:

The possible paths and the corresponding path lengths are as follows

TABLE 1 POSSIBLE PATH AND ITS LENGTH

\begin{tabular}{|c|l|l|l|}
\hline $\mathrm{i}$ & Path $\mathrm{P}_{\mathrm{i}}$ & $\begin{array}{l}\text { Member } \\
\text { ship } \\
\text { Function } \mu_{\mathrm{i}}\end{array}$ & $\begin{array}{l}\text { Non-membership } \\
\text { Function } \gamma_{\mathrm{i}}\end{array}$ \\
\hline 1 & $\begin{array}{l}1 \rightarrow 4 \rightarrow \\
5 \rightarrow 6\end{array}$ & $\mathrm{~L}_{\mu 1}=(6,13,18 ; 1)$ & $\mathrm{L}_{\gamma 1}=(10,17,21 ;)$ \\
\hline 2 & $\begin{array}{l}1 \rightarrow 2 \rightarrow \\
3 \rightarrow 6\end{array}$ & $\mathrm{~L}_{\mu 2}=(8,17,24 ; 1)$ & $\mathrm{L}_{\gamma 2}=(12,21,26 ;)$ \\
\hline 3 & $\begin{array}{l}1 \rightarrow 2 \rightarrow 4 \\
5 \rightarrow 6\end{array}$ & $\mathrm{~L}_{\mu 3}=(12,19,281)$ & $\mathrm{L}_{\gamma 3}=(19,24,31 ;)$ \\
\hline 4 & $\begin{array}{l}1 \rightarrow 2 \rightarrow 3 \\
5 \rightarrow 6\end{array}$ & $\mathrm{~L}_{\mu 4}=(9,20,28 ; 1)$ & $\mathrm{L}_{\gamma 4}=(17,25,321)$ \\
\hline
\end{tabular}

Step 3:

$$
\mathrm{L}_{\text {min }}=\{6,20,18 ; 1\} \quad \text { and } \mathrm{L}_{\max }=\{19,17,32 ; 1\}
$$

\section{Step 4}

\section{TABLE 2 RESULTS OF THE NETWORK BASED ON} LEVEL $\lambda$ - TLR WEIGHTED INDEX

\begin{tabular}{|c|l|c|c|c|c|}
\hline $\mathbf{i}$ & Path $\mathrm{P}_{\mathrm{i}}$ & $\begin{array}{l}\mathrm{W}_{\mathrm{A}} \\
\left(\begin{array}{l}\left.\mathrm{L}_{\min }, \mathrm{L}_{\mu \mathrm{i}}\right)\end{array}\right.\end{array}$ & $\begin{array}{c}\mathbf{R} \\
\mathbf{a} \\
\mathbf{n} \\
\mathbf{k}\end{array}$ & $\begin{array}{l}\mathrm{W}_{\mathrm{A}} \\
\left(\begin{array}{l}\mathrm{L}_{\max }, \mathrm{L}_{\gamma \mathrm{i}}\end{array}\right)\end{array}$ & $\begin{array}{c}\mathbf{a} \\
\mathbf{n} \\
\mathbf{k}\end{array}$ \\
\hline $\mathbf{1}$ & $\begin{array}{l}1 \rightarrow 4 \rightarrow \\
5 \rightarrow 6\end{array}$ & $\mathbf{6}$ & $\mathbf{1}$ & $\mathbf{1 4 . 5}$ & $\mathbf{1}$ \\
\hline $\mathbf{2}$ & $\begin{array}{l}1 \rightarrow 2 \rightarrow \\
3 \rightarrow 6\end{array}$ & $\mathbf{7}$ & $\mathbf{2}$ & $\mathbf{1 5 . 5}$ & $\mathbf{2}$ \\
\hline $\mathbf{3}$ & $\begin{array}{l}1 \rightarrow 2 \rightarrow 4 \\
5 \rightarrow 6\end{array}$ & $\mathbf{9}$ & $\mathbf{4}$ & $\mathbf{1 9}$ & $\mathbf{4}$ \\
\hline $\mathbf{4}$ & $\begin{array}{l}1 \rightarrow 2 \rightarrow 3 \\
5 \rightarrow 6\end{array}$ & $\mathbf{7 . 5}$ & $\mathbf{3}$ & $\mathbf{1 8}$ & $\mathbf{3}$ \\
\hline
\end{tabular}


TABLE 3 RESULTS OF THE NETWORK BASED ON LEVEL $\lambda$ - TLR MEAN INDEX

\begin{tabular}{|c|c|c|c|c|c|}
\hline$\overline{\mathbf{i}}$ & Path $\mathrm{P}_{\mathrm{i}}$ & $T_{\min }\left(L_{\mu i}\right)$ & $\begin{array}{l}\mathrm{R} \\
\mathrm{a} \\
\mathrm{n} \\
\mathrm{k}\end{array}$ & $T_{\max }\left(L_{\gamma i}\right)$ & $\begin{array}{l}\text { R } \\
\text { a } \\
\text { n } \\
\text { k }\end{array}$ \\
\hline 1 & $\begin{array}{l}1 \rightarrow 4 \rightarrow \\
5 \rightarrow 6\end{array}$ & 8.5 & 1 & 8 & 1 \\
\hline 2 & $\begin{array}{l}1 \rightarrow 2 \rightarrow \\
3 \rightarrow 6\end{array}$ & 11.5 & 2 & 9.5 & 2 \\
\hline 3 & $\begin{array}{l}1 \rightarrow 2 \rightarrow 4 \\
5 \rightarrow 6\end{array}$ & 16.5 & 4 & 15.5 & 4 \\
\hline 4 & $\begin{array}{l}1 \rightarrow 2 \rightarrow 3 \\
5 \rightarrow 6\end{array}$ & 13 & 3 & 13.5 & 3 \\
\hline
\end{tabular}

TABLE 4 RESULTS OF THE NETWORK BASED ON LEVEL $\lambda$ - TLR CENTROID INDEX

\begin{tabular}{|c|l|c|c|c|c|}
\hline $\mathbf{i}$ & Path $\mathrm{P}_{\mathrm{i}}$ & $\mathrm{T}_{\text {centroid }}\left(\mathrm{L}_{\mu \mathrm{i}}\right.$ & $\begin{array}{c}\mathbf{R} \\
\mathbf{a} \\
\mathbf{n} \\
\mathbf{k}\end{array}$ & $\mathrm{T}_{\text {centroid }}\left(\mathrm{L}_{\gamma \mathrm{i}}\right)$ & $\begin{array}{c}\mathbf{R} \\
\mathbf{a} \\
\mathbf{n} \\
\mathbf{k}\end{array}$ \\
\hline $\mathbf{1}$ & $\begin{array}{l}1 \rightarrow 4 \rightarrow \\
5 \rightarrow 6\end{array}$ & $\mathbf{7 . 6}$ & $\mathbf{1}$ & $\mathbf{8 . 6 6}$ & $\mathbf{1}$ \\
\hline $\mathbf{2}$ & $\begin{array}{l}1 \rightarrow 2 \rightarrow \\
3 \rightarrow 6\end{array}$ & $\mathbf{1 0 . 3}$ & $\mathbf{2}$ & $\mathbf{1 0 . 3}$ & $\mathbf{2}$ \\
\hline $\mathbf{3}$ & $\begin{array}{l}1 \rightarrow 2 \rightarrow 4 \\
5 \rightarrow 6\end{array}$ & $\mathbf{1 5}$ & $\mathbf{4}$ & $\mathbf{1 6 . 6}$ & $\mathbf{4}$ \\
\hline $\mathbf{4}$ & $\begin{array}{l}1 \rightarrow 2 \rightarrow 3 \\
5 \rightarrow 6\end{array}$ & $\mathbf{1 1 . 6 6}$ & $\mathbf{3}$ & $\mathbf{1 4 . 6 6}$ & $\mathbf{3}$ \\
\hline
\end{tabular}

TABLE 5 RESULTS OF THE NETWORK BASED ON LEVEL $\lambda$ - TLR SIGNED INDEX

\begin{tabular}{|c|l|c|c|c|c|c|}
\hline $\mathbf{i}$ & Path $\mathrm{P}_{\mathrm{i}}$ & $\mathrm{d}\left(\mathrm{L}_{\mu \mathrm{i}}, 0\right)$ & $\begin{array}{c}\mathbf{R} \\
\mathbf{a} \\
\mathbf{n}\end{array}$ & $\mathrm{d}\left(\mathrm{L}_{\gamma \mathrm{i}}, 0\right)$ & $\begin{array}{l}\mathbf{R} \\
\mathbf{a} \\
\mathbf{n}\end{array}$ \\
\hline $\mathbf{1}$ & $\begin{array}{l}1 \rightarrow 4 \rightarrow \\
5 \rightarrow 6\end{array}$ & $\mathbf{7 . 3}$ & $\mathbf{1}$ & $\mathbf{4}$ & $\mathbf{1}$ \\
$\mathbf{2}$ & & & & \\
\hline $\mathbf{2}$ & $\begin{array}{l}1 \rightarrow 2 \rightarrow \\
3 \rightarrow 6\end{array}$ & $\mathbf{9 . 8}$ & $\mathbf{2}$ & $\mathbf{5}$ & $\mathbf{2}$ \\
\hline $\mathbf{3}$ & $\begin{array}{l}1 \rightarrow 2 \rightarrow 4 \\
5 \rightarrow 6\end{array}$ & $\mathbf{1 4 . 3}$ & $\mathbf{4}$ & $\mathbf{7 . 8}$ & $\mathbf{4}$ \\
\hline $\mathbf{4}$ & $\begin{array}{l}1 \rightarrow 2 \rightarrow 3 \\
5 \rightarrow 6\end{array}$ & $\mathbf{1 1}$ & $\mathbf{3}$ & \multicolumn{2}{|c|}{$\mathbf{6 . 8}$} & $\mathbf{3}$ \\
\hline
\end{tabular}

Step 5 Hence Path $\mathrm{P}_{1}: 1 \rightarrow 4 \rightarrow 5 \rightarrow 6$ is the Intuitionistic fuzzy shortest path since it has the minimum level $\lambda$ - TLR in weighted average Index, Mean Index,

Centroid Index, also Signed distance Index and the corresponding shortest path length is

$\mathrm{L}_{\mu 1}=(6,13,18 ; 1)_{\mathrm{LR}}$ and $\mathrm{L}_{\gamma 1}=(10,17,21 ;)_{\mathrm{LR}}$

Hence we find that the solution which is obtained for intuitionistic fuzzy shortest path problem . For verifying the results we have three different new algorithms to find the shortest path in Intuitionistic fuzzy numbers.

\section{A COMPARATIVE STUDY}

A comparative study on solving the network problem using TLR Intuitionistic Fuzzy Number present the following new methods, evolved the process of the research.

The four methods $\lambda$-TLR Weighted Index, $\lambda$-TLR Mean Index, $\lambda$-TLR centroid Index,$\lambda$-TLR Signed Index presented in the paper. The Application of all the possible path in the evolved four methods brings forth the same results. Thus we strongly concluded that, any one method can be adopted for deriving the results.

\section{COMPARISON WITH EXISTING ALGORITHM}

In the process of finding the shortest path ,the research findings evolved different methods of obtaining the Shortest path using (i) Arc Length (ii) Using Similarity Approach(iii)Using $\lambda$-TLR Weighted Index, $\lambda$-TLR Mean Index, $\lambda-$ TLR centroid Index and $\lambda-$ TLR Signed Index.

This paper presents the last approach (iii) involved in finding the shortest path using LR type Triangular fuzzy number.The previous two methods were already discussed in the paper titled " on solving network problems using new algorithm with Intuitionistic Fuzzy arc length" published in international conference on Mathematics in Engineering and Business Management. And Second one "Using similarity Degree Approach to find Shortest path for Intuitionistic Fuzzy in a network" in a referred international journal.

\section{CONCLUSION}

The Algorithm evolved in the process of finding the shortest path problem found to be fruitful. As the study brought forth three algorithm with the same results.In this paper we developed three algorithms for solving shortest path problem on a network., where the shortest path is identified using the concept of ranking function. Decision maker can choose the best path among various alternative methods from the list of ranking. It helps to conclude that the new algorithms developed in the current paper is an alternative and improved form of previous methods to get Shortest path. Hence we 
conclude that the algorithms developed in the current research are the simplest and is the alternative method for getting the shortest path in Intuitionistic fuzzy.

\section{REFERENCES}

[1] D.Dubois and H.Prade,"Fuzzy Sets and Systems",Academic Press, New York,1980.

[2] K.Atanassov, Intuitionistic Fuzzy Sets and Systems, volume 20, No. $187-961986$.

[3] Kung J.Y.,Chuang T.N, and C.T.Lin ,Decision Making On Network Problem With Fuzzy arc lengths, IMACS Multiconference on Computational Engineering in Systems Applications (CESA578580)2006

[4] Okada S.Soper.T.,"A Shortest Path Problem on a Network With Fuzzy arcLengths". Fuzzy Sets and Systems 109,129-140,2000.

[5] L.Sujatha, "Fuzzy shortest path problem based on T LR type fuzzy number using acceptability index," International Journal of Engineering and Technology, 6(2009),575-578.

[6] Klein,C.M 1991. Fuzzy Shortest Paths, Fuzzy Sets And Systems 39,27- 41, 2011

[7] Nayeem, S.M.A and pal M., 'Shortest Path problem on a network with imprecise edge weight" ,Fuzzy Optimization and Decision Making, Vol.,4 No.4., pp293-312,2005

[8] S.Elizabath and L.Sujatja, "Fuzzy Shortest Path Problem Based on Index Ranking " Journal of Mathematics Research vol3,N0.4 . November 2011.

[9] X. Entropy, ,Length Measure and Similarity Measure of Fuzzy Sets and their Relations, Fuzzy sets and systems,52.305 -18, [13] 1992.

[10] Iraj Mahadavi,Ali Tajdin,Rahele Nourifar "Using Fuzzy Optimization Approach for Shortest Path Problem in Network flow".

[11] Odada S., Gen M., "Fuzzy shortest path problems".Computer and Industrial engineering, 27,465 468,1994 ..

[12] A.Nagoor gani,M.Mohammed Jabbarulla " Onsearching Intuitionistic Fuzzy Shortest path in a Network ".Applied Mathematical Sciences, Vol 4, 2010,no,69,3447-3454.

[13]Okada, S.,(2004) Fuzzy shortest path problem incorporating interactivity Among paths, Fuzzy Sets and Systems, 142 ,335-357.
[14] Lin K.C.,Chern M.S., The Fuzzy Shortest Path Problem and its Most Liu Vital arcs., Fuzzy Sets and Systems58, $343-$ Systems 58,343-353,1993.

[15] S.Elizabeth and L.Sujatha, "Fuzzy Shortest Path Promblem Based on Level $\lambda$ - LR Type Representation of Fuzzy Intervel:International conference on Mathematics in Engineering and Business management 978-81-8286-015-5

[16] WJ. New Similarity Measures on Fuzzy Sets and On Elements. Fuzzy Sets and Systems., 85, 305-9, 1997.

[17] Kiran Yadav and Ranjit Biswas "An Approach to Find k th Shortest Path Using Fuzzy Logic"., Internation Journal of Computational Cognition vol8 no.1.March 2010.

[18] Zadeh .L.A Fuzzy sets., Inform.Control. Vol 8 pages.338-353 1965 .

[19] Zadeh L.A 'Fuzzy sets as a basis for a theory of possibility",Fuzzy Sets and Systems,Vol 1, No 1,pp328,1978 .

[20] Yu, J.R and Wei , T.H "Solving the fuzzy shortest path problem by using a linear multiple objective programming", Journal of the Chiness institute of Industrial Engineers, Vol .,24,pp.360-365,2007.

[21] Sudhir, K.P and Rimple, P., Fuzzy sets and Their Applications, Pragato \{rakashan, Meerut, India, First Edition, Sujatha ,L. and Sattanathan ,R. "Fuzzy Shortest path problem based on similarity degree using LR type fuzzy numbers" , International Journal of Computational Intelligence Research and Applications, Vol .3, No. 2, pp.213-216,2009.

[22] Sujatha, L. and Sattanathan ,R., "Fuzzy Shortest path problem based on interval numbers", International Journal of Fuzzy Systems and Rough Systems, Vol 2., N0.2 pp 57-60, 2009.

[23] P.Jayagowri and Dr.G.Geetharamani .," Using similarity degree Approach to find Shortest Path for Intuitionsitc Fuzzy in a Network" International conference on computing communication and Applications Feb 2012 .,paper published in IEEE journal 2012.

[24] P.Jayagowri and Dr.G.Geetharamani.," On solving network problems using new algorithm with Intuitionstic fuzzy arc length:International conference on Mathematics in Engineering and Business management., proceedings March 2012 